\title{
Huge enhancement of backward second-harmonic generation with slow light in photonic crystals
}

\author{
Rumen Iliew, ${ }^{1,2, *}$ Christoph Etrich, ${ }^{3}$ Thomas Pertsch, ${ }^{3}$ Falk Lederer, ${ }^{2}$ and Yuri S. Kivshar ${ }^{1}$ \\ ${ }^{1}$ Nonlinear Physics Center, Research School of Physics and Engineering, The Australian National University, Canberra ACT 0200, Australia \\ ${ }^{2}$ Institute of Condensed Matter Theory and Solid State Optics, Friedrich-Schiller-Universität Jena, \\ Max-Wien-Platz, 1, 07743 Jena, Germany \\ ${ }^{3}$ Institute of Applied Physics/Ultra Optics, Friedrich-Schiller-Universität Jena, Max-Wien-Platz 1, 07743 Jena, Germany
}

(Received 4 December 2009; published 19 February 2010)

\begin{abstract}
We study theoretically forward and backward second-harmonic generation in a two-dimensional photonic crystal structure made of lithium niobate. The aim of this article is twofold: First, we propose a reliable modal algorithm for describing the light propagation taking into account the vectorial character of the interacting fields as well as the tensorial character of the nonlinearity and verify it by means of the nonlinear finite-difference time-domain method. Second, we propose a photonic crystal where we obtain a giant efficiency increase for backward second-harmonic generation with slow light.
\end{abstract}

DOI: 10.1103/PhysRevA.81.023820

PACS number(s): 42.70.Qs, 42.65.Ky, 42.70.Mp

\section{INTRODUCTION}

Since the early days of nonlinear optics [1] efficient second-harmonic generation (SHG) has been a subject of continuous scientific interest. Soon the crucial role of the linear phase mismatch between the interacting waves for the SHG efficiency was revealed [2]. In general, the phase mismatch in a three-wave mixing process $\omega_{1}+\omega_{2}=\omega_{3}$ is defined through the wave vector difference $\Delta \mathbf{k}=\mathbf{k}_{3}-\mathbf{k}_{1}-\mathbf{k}_{2}$ of the three interacting plane waves. If two of these waves coincide and are indistiguishable (same frequency $\omega_{1}=\omega_{2}=\omega_{3} / 2$ and same polarization or modal fields) this process is called type I SHG, in contrast to type II SHG, where still these two so-called fundamental harmonis (FHs) at $\omega_{1}=\omega_{2}$ can be distinguished (different polarization, different modes). It was shown that for the degenerate case of type I SHG (only two interacting waves) with plane waves in a bulk medium optimal generation of a second-harmonic $(\mathrm{SH})$ field from a strong (pump) fundamental harmonic field is achieved, if the phase matching condition $\mathbf{k}_{\mathrm{s}}=2 \mathbf{k}_{\mathrm{f}}$ involving the wave vectors of the two interacting waves (s...SH, f...FH) is fulfilled. Then, the two wave vectors are obviously parallel, which is called collinear propagation. Due to material dispersion, in homogeneous media the phase matching condition for collinear propagation is usually not fulfilled. However, the anisotropy of the common nonlinear crystals can be utilized to obtain phase matching between waves of different polarization (ordinary, extraordinary in uniaxial crystals or more complex for biaxial crystals) and for certain directions of propagation relative to the crystal's principal axes. These directions of polarization and light propagation given by the phase matching condition can substantially reduce the effective nonlinear coefficient due to its tensorial character. However, for certain crystals, e.g., lithium niobate $\left(\mathrm{LiNbO}_{3}\right)$, the larger diagonal nonlinear coefficient can be used when quasi-phase matching (QPM) [2-4] is achieved by poling the nonlinear material with a certain periodicity, usually given by the so-called coherence length $L_{\mathrm{c}}$ which depends on the dispersion relations of the

*rumen.iliew@uni-jena.de participating waves. The dielectric is still homogeneous as far as its linear properties are concerned.

Usually, in SHG the generation of a forward traveling SH wave (same direction as the FH) comes to mind, as this is historically most widely applied and phase matching can be achieved even in homogeneous crystals. More specifically, in forward SHG, the directions of energy transport of the interacting (plane) waves are in the same direction (angle of up to $90^{\circ}$ ). Backward parametric three wave interactions utilizing strongly birefringent crystals were proposed early [5] but experimental verification remained a challenge. Using QPM with shorter poling periods or higher-order QPM [6-8], the generation of a backward traveling $\mathrm{SH}$ wave is also experimentally feasible $[9,10]$. In these backward three-wave mixing schemes the dynamics of light propagation fundamentally differs $[6,8,11,12]$ from the forward case. One interesting aspect releated to the peculiar dynamics of backward threewave interactions is the possibility of obtaining a mirrorless optical parametric oscillator, as the idler wave is generated in opposite direction to signal and pump waves leading effectively to a reflection of the $\mathrm{FH}$ into $\mathrm{SH}[5,11,13]$. The same phenomenon is used for nonlinear localization [14]. Another interesting application of backward second-harmonic generation (BSHG) is pulse shaping [15]. BSHG can lead to self-pulsations in linearly resonant [16] and in nonresonant configurations [17] for a continuous-wave input. However, as poling of the nonlinear material at subwavelength scale to obtain fundamental order QPM for BSHG is hardly achievable, also the possibility of tailoring the properties of the linear modes via periodic structuring to get phase matching is an important option.

The possibility of achieving phase matching for forward SHG via an one-dimensional (1D) periodic modulation of the linear properties of the medium was proposed [18-20] and experimentally verified [21] decades ago. Due to the recent interest in the field of photonic crystals phase matching via an $1 \mathrm{D}$ periodic dielectric modulation regained scientific attention. In addition to providing phase matching, these structured materials also allow for a novel means of further increasing the conversion efficiency, relying on the small group velocity (and consequently the large density of states) near band edges [22-25] in finite-length 1D gratings and 
defects. The linear effects originating from the finiteness of the periodic structure, as reflection at boundaries and transmission resonances, which influence the nonlinear propagation [25] are not considered in this work which will be dealing with structures that are truly periodic and hence, infinite along propagation direction regarding the linear properties.

Meanwhile, the enhancement of nonlinear effects via slow light in two- and three-dimensional (3D) photonic crystals (PhCs) was proposed [26-29]. An efficiency increase for direct third-harmonic generation not known before was experimentally achieved with slow light in a line defect waveguide in a silicon PhC membrane with third-order nonlinearity [30]. With the advances in nanostructure technology a high-contrast two-dimensional (2D) pattering on the submicrometer scale of quadratically nonlinear materials became possible [31-33]. More than a decade ago, sum-frequency generation and phase matching in a $2 \mathrm{D}$ photonic crystal were investigated using a Green's function approach [34] in the undepleted pump approximation (UDPA). There, many more degrees of freedom (structure and propagation direction) for achieving phase matching exist. However, the approach presented cannot properly reproduce the results for a homogeneous medium in the UDPA and naturally fails if a substantial fraction of energy is converted to the SH field. Furthermore, many nonlinear phenomena rely on the backaction of the generated $\mathrm{SH}$ on the $\mathrm{FH}$, as temporal and spatial solitons or optical bistability in resonators. These phenomena, essential for all-optical routing and switching, cannot be described by UDPA. Later, coupled equations of motion for slowly varying envelopes were obtained using a multiscale analysis starting from the wave equation for the electric field [35]. Here, enabled by the peculiar dispersion relation of the $\mathrm{PhC}$, three-wave mixing employing a backward wave was investigated. The case of non-phase matched interaction was not considered, however. A modal description of forward and backward collinear secondharmonic generation in PhCs including phase mismatch terms was proposed and compared to results from nonlinear finitedifference time-domain (FDTD) calculations for the forward case in 2D PhCs recently [36], clearly illustrating the influence of phase mismatch and material dispersion as well as the efficiency enhancement via slow light. The enhancement of SHG in PhC slabs was theoretically [37] and experimentally [38] investigated by means of varying angle transmission and reflection spectroscopy. There, however, the enhancement essentially stems from the resonant cavity (the slab) and lattice induced coupling to these states, whereas in the present article we are interested in the propagation effects in the plane of the classical SHG.

Phase matched (anti-)collinear BSHG was obtained almost independently of the direction of propagation in a properly designed 2D PhC [39]. However, the employed numerical scheme based on a multiple-scattering method relies on the UDPA. A coherence length larger than 1,200 $\mu \mathrm{m}$ for a large range of propagation directions was proposed. As the goal did not consist in enhancing the efficiency for one particular direction but rather phase matching for any direction of light propagation slow light was not utilized in this design. In a new design including silver omnidirectional phase matched BSHG was used to obtain a nonlinear metamaterial lens [40] for focusing of the $\mathrm{SH}$. Recently, a 2D PhC design was theoretically proposed where SHG is phase matched over a wide angular range in the subdiffractive region of the dispersion relation, allowing for efficient interaction of narrow beams [41].

A second application of 2D quadratically nonlinear $\mathrm{PhCs}$ was the utilization of strong linear light localization at point defects within the band gap of the crystal, where generation of terahertz radiation via optical rectification [42], SHG $[43,44]$, and optical parametric oscillators $[45,46]$ were proposed theoretically.

In this article we propose a $2 \mathrm{D} \mathrm{PhC}$ structure in $\mathrm{LiNbO}_{3}$ where phase matched forward as well as backward collinear type I SHG is possible, however, at different wavelengths. We are not interested in transverse spatial effects and, hence, assume the beams to be sufficiently broad in the transverse dimension. Previous investigations did not consider the power efficiency of the conversion process but merely demonstrated the possibility of achieving phase matching. From a modal approach we derive explicit expressions relating the generated $\mathrm{SH}$ power to the input $\mathrm{FH}$ power. We show that for properly designed PhCs SHG efficiencies for forward and backward SHG can be achieved exceeding those in a homogeneous phase matched medium. We verify the dynamics obtained from our modal approach with rigorous calculations employing the $2 \mathrm{D}$ FDTD method.

In Sec. II we formulate the modal equations describing the dynamics of the guided power, which is the most appropriate description of experimental setups because one can directly compare the results with those in different other systems, as, e.g., with waveguide or plane wave geometries. In contrast to many other proposals a primary goal of our work consists in providing an explicit quantitative analysis of the nonlinear process, based on the model theory. This is verified by rigorous calculations. We introduce a nonlinear figure of merit that allows a direct characterization of the strength of the nonlinear interaction in this $2 \mathrm{D} \mathrm{PhC}$, independent of the pump power or the propagation length. In the following section the $\mathrm{PhC}$ structure is introduced and the phase matching curves are discussed. Inspecting the field distribution we reveal that forward SHG as well as BSHG are feasible. By using the modal approach we discuss the efficiencies and propose an improved design for highly efficient BSHG. In Sec. IV we present results of the modeling with the nonlinear FDTD method. We discuss the simulations with respect to numerical stability and numerical dispersion. Finally, we conclude the article.

\section{MODAL DESCRIPTION OF COLLINEAR SHG}

In the following we generalize the modal description of light propagation in PhCs given in Ref. [36] toward the tensorial character of nonlinear interactions and derive a figure of merit (FOM) for their efficiency.

We are interested only in spatial and temporal effects along the propagation direction, in the transverse direction $[\perp \equiv(x, y)]$ we assume plane wave excitation according to the shape of the (linear) normal modes of the $\mathrm{PhC}$, which are Bloch waves. Then all dynamics of the interaction will develop in the propagation direction $z$. Ideally, this will be the direction of energy transport of both participating Bloch waves. Again, 
in the Fourier domain we depart from the conjugated form of Lorentz' reciprocity theorem [47] to obtain evolution equations for the envelopes. The theorem states that for two electromagnetic fields $\left(\mathbf{E}_{1}, \mathbf{H}_{1}\right)$ and $\left(\mathbf{E}_{2}, \mathbf{H}_{2}\right)$ inducing the respective dielectric polarizations $\mathbf{P}_{1}=\varepsilon_{0} \varepsilon_{1}(\mathbf{r}, \omega) \mathbf{E}_{1}(\mathbf{r}, \omega)$ and $\mathbf{P}_{2}=\varepsilon_{0} \varepsilon_{1}(\mathbf{r}, \omega) \mathbf{E}_{2}(\mathbf{r}, \omega)+\mathbf{P}_{\mathrm{p}}(\mathbf{r}, \omega)$ and satisfying Maxwell's equations we have for nonabsorbing materials

$$
\int_{\partial V} \mathrm{~d} \mathbf{A} \cdot\left[\mathbf{E}_{1}^{*}(\mathbf{r}, \omega) \times \mathbf{H}_{2}-\mathbf{H}_{1}^{*} \times \mathbf{E}_{2}\right]=\imath \omega \int_{V} \mathrm{~d}^{3} \mathbf{r} \mathbf{P}_{\mathrm{p}} \cdot \mathbf{E}_{1}^{*},
$$

where $\partial V$ is the boundary of the (arbitrary) volume $V$. The polarization $\mathbf{P}_{1}$ is assumed to be linear to facilitate the exact solution of the (unperturbed) problem $\left(\varepsilon_{1}, \mathbf{E}_{1}, \mathbf{H}_{1}\right)$. For system 2 we allow for a nonlinear perturbative polarization $\mathbf{P}_{\mathrm{p}}$. In particular, we use the modal field of the Bloch waves of the fundamental and of the second harmonic

$$
\begin{gathered}
\mathbf{E}_{1}(\mathbf{r}, \omega)=\mathbf{e}_{n_{\mathrm{f}, \mathrm{s}} \mathbf{k}_{\mathrm{f}, \mathrm{s}}}^{\mathrm{f}, \mathrm{r}}(\mathbf{r}) \exp \left(l \mathbf{k}_{n_{\mathrm{f}, \mathrm{s}} \perp} \cdot \mathbf{r}_{\perp}\right) \exp \left[\imath k_{n_{\mathrm{f}, \mathrm{s}} z}(\omega) z\right] \\
\mathbf{H}_{1}(\mathbf{r}, \omega)=\mathbf{h}_{n_{\mathrm{f}, \mathrm{s}} \mathrm{f} \mathbf{k}_{\mathrm{f}, \mathrm{s}}}(\mathbf{r}) \exp \left(l \mathbf{k}_{n_{\mathrm{f}, \mathrm{s}} \perp} \cdot \mathbf{r}_{\perp}\right) \exp \left[l k_{n_{\mathrm{f}, \mathrm{s}} z}(\omega) z\right],
\end{gathered}
$$

where $\mathbf{e}_{n_{\mathrm{f}} \mathbf{k}_{\mathrm{f}}}^{\mathrm{f}}, \mathbf{h}_{n_{\mathrm{f}} \mathbf{k}_{\mathrm{f}}}^{\mathrm{f}}, \mathbf{e}_{n_{\mathrm{s}} \mathbf{k}_{\mathrm{s}}}^{\mathrm{s}}$, and $\mathbf{h}_{n_{\mathrm{s}} \mathbf{k}_{\mathrm{s}}}^{\mathrm{s}}$ denote the lattice-periodic electric and magnetic Bloch amplitudes of the fundamental (index f) and second-harmonic (index s) waves with corresponding Bloch vectors $\mathbf{k}_{\mathrm{f}}=\left(\mathbf{k}_{n_{\mathrm{f}} \perp}, k_{n_{\mathrm{f}} z}\right), \mathbf{k}_{\mathrm{s}}=\left(\mathbf{k}_{n_{\mathrm{s}} \perp}, k_{n_{\mathrm{s}} z}\right)$ in the respective bands with indices $n_{\mathrm{f}}$ and $n_{\mathrm{s}}$. As we are interested in type I SHG we assume that only one Bloch mode contributes both to the $\mathrm{FH}$ and $\mathrm{SH}$, respectively. Usually this is satisfied if the different bands are well separated in frequency or via different propagation direction. However, in certain cases it may be necessary to include more modes into the analysis. The dispersion relation $\omega=\omega_{n}(\mathbf{k})$ gives the relation between Bloch vector and frequency. Later we will require a transverse phase matching condition and a strictly periodic transverse behavior resulting in constant transverse Bloch vectors $\mathbf{k}_{n_{\mathrm{f}, \mathrm{s}} \perp}$. Then, we can use the inverted dispersion relation $k_{z}\left(\omega ; \mathbf{k}_{\perp}\right)$ and drop the argument $\mathbf{k}_{\perp}$ as this quantity is a fixed parameter. In this case the Bloch amplitudes can be denoted by $\mathbf{e}_{\mathrm{f}, \mathrm{s}}(\mathbf{r}, \omega), \mathbf{h}_{\mathrm{f}, \mathrm{s}}(\mathbf{r}, \omega)$ with an explicit frequency dependence. However, we restrict the bandwidth of the investigated processes to very narrow regions around $\omega_{0}$ and $2 \omega_{0}$ and, hence, assume that the frequency dependence of the Bloch amplitudes can be neglected, whereas the dispersion of the Bloch vector is kept.

Consequently, the ansatz for the perturbed problem $\left(\varepsilon_{1}, \mathbf{E}_{2}, \mathbf{H}_{2}, \mathbf{P}_{\mathrm{p}}\right)$ in the time domain is

$$
\begin{aligned}
\left(\begin{array}{c}
\mathbf{E}_{2}(\mathbf{r}, t) \\
\mathbf{H}_{2}(\mathbf{r}, t)
\end{array}\right)= & \frac{1}{2} a(z, t)\left(\begin{array}{c}
\mathbf{e}_{\mathrm{f}}\left(\mathbf{r}, \omega_{0}\right) \\
\mathbf{h}_{\mathrm{f}}\left(\mathbf{r}, \omega_{0}\right)
\end{array}\right) \exp \left(\imath \mathbf{k}_{n_{\mathrm{f}} \perp} \cdot \mathbf{r}_{\perp}\right) \exp \left(\imath k_{\mathrm{f} z}^{0} z\right) \\
& \times \exp \left(-\imath \omega_{0} t\right)+\frac{1}{2} b(z, t)\left(\begin{array}{c}
\mathbf{e}_{\mathrm{s}}\left(\mathbf{r}, 2 \omega_{0}\right) \\
\mathbf{h}_{\mathrm{s}}\left(\mathbf{r}, 2 \omega_{0}\right)
\end{array}\right) \\
& \times \exp \left(\imath \mathbf{k}_{n_{\mathrm{s}} \perp} \cdot \mathbf{r}_{\perp}\right) \exp \left(\imath k_{\mathrm{s} z}^{0} z\right) \\
& \times \exp \left(-2 \imath \omega_{0} t\right)+(*) \\
\mathbf{P}_{\mathrm{p}}(\mathbf{r}, t)= & \frac{1}{2}\left[\mathbf{p}_{\mathrm{f}}(\mathbf{r}, t) \exp \left(\imath \mathbf{k}_{n_{\mathrm{f}} \perp} \cdot \mathbf{r}_{\perp}\right) \exp \left(\imath k_{\mathrm{f} z}^{0} z\right) \exp \left(-\imath \omega_{0} t\right)\right. \\
+ & \mathbf{p}_{\mathrm{s}}(\mathbf{r}, t) \exp \left(\imath \mathbf{k}_{n_{\mathrm{s}} \perp} \cdot \mathbf{r}_{\perp}\right) \exp \left(\imath k_{\mathrm{s} z}^{0} z\right) \\
& \left.\times \exp \left(-2 \imath \omega_{0} t\right)\right]+(*)
\end{aligned}
$$

where $k_{\mathrm{f} z}^{0}=k_{\mathrm{f} z}\left(\omega_{0}\right)$ and $k_{\mathrm{s} z}^{0}=k_{\mathrm{s} z}\left(2 \omega_{0}\right)$ are the central Bloch vectors, $a$ and $b$ the slowly varying envelopes of the fundamental and the second harmonic wave, respectively, and $\mathbf{p}_{\mathrm{f}}$ and $\mathbf{p}_{\mathrm{s}}$ are the slowly (in $t$ ) varying nonlinear polarizations at $\omega_{0}$ and $2 \omega_{0}$, respectively. In the following a periodicity of the structure in the $z$ direction with period $L$ is assumed. The volume $V$ is chosen to be the respective unit cell $\Omega$ centered at pertinent $z$. This corresponds to a separation into a fast $z$ dependence (fast oscillations on the scale of $\mathrm{PhC}$ period, Bloch amplitudes), which is spatially integrated over, and a slow $z$ dependence, which changes slowly over many periods and can be moved out of the integrals. Inserting the Fourier transform of (4) into (1), performing the integration along the boundaries in propagation direction, and assuming sufficiently small changes over $L$ in propagation direction, we obtain by means of the rotating wave approximation differential equations for the two field envelopes coupled via the perturbative nonlinear polarization. Accounting for Kleinman symmetry the two contributions of the nonlinear polarization are

$$
\begin{aligned}
\mathbf{p}_{\mathrm{f}}= & 2 \varepsilon_{0} a^{*} b \hat{d}\left(-\omega_{0} ;-\omega_{0}, 2 \omega_{0}\right):\left(\mathbf{e}_{\mathrm{f}}^{*} \mathbf{e}_{\mathrm{s}}\right) \\
& \times \exp \left[\imath\left(\mathbf{k}_{n_{\mathrm{s}} \perp}-2 \mathbf{k}_{n_{\mathrm{f}} \perp}\right) \cdot \mathbf{r}_{\perp}\right] \exp \left[\imath\left(k_{\mathrm{s} z}^{0}-2 k_{\mathrm{f} z}^{0}\right) z\right] \\
\mathbf{p}_{\mathrm{s}}= & \varepsilon_{0} a^{2} \hat{d}\left(-2 \omega_{0} ; \omega_{0}, \omega_{0}\right):\left(\mathbf{e}_{\mathrm{f}} \mathbf{e}_{\mathrm{f}}\right) \\
& \times \exp \left[-\imath\left(\mathbf{k}_{n_{\mathrm{s}} \perp}-2 \mathbf{k}_{n_{\mathrm{f}} \perp}\right) \cdot \mathbf{r}_{\perp}\right] \exp \left[-\imath\left(k_{\mathrm{s} z}^{0}-2 k_{\mathrm{f} z}^{0}\right) z\right] .
\end{aligned}
$$

Here we adopted the notation of Ref. [48] with the contracted $\hat{d}$ tensor and the six-component second-order column vector of the fields $\left(\mathbf{e}_{1} \mathbf{e}_{2}\right)$ giving the polarization vector $K \varepsilon_{0} \hat{d}:\left(\mathbf{e}_{1} \mathbf{e}_{2}\right)$ where $K=1$ for degenerate waves $\mathbf{e}_{1}$ and $\mathbf{e}_{2}$ and $K=2$ otherwise. Inserting (6) and (7) into the differential equations we obtain after some approximations (see Ref. [36])

$$
\begin{aligned}
& l \frac{\partial a}{\partial z}+\frac{l}{v_{\mathrm{gf}}} \frac{\partial a}{\partial t}+b a^{*} \exp (\iota \Delta k z) \frac{\omega_{0} \varepsilon_{0}}{2 L \tilde{P}_{\mathrm{f}}} \int_{\Omega} \mathrm{d}^{3} \mathbf{r} \times \mathbf{e}_{\mathrm{f}}^{*} \cdot\left[\hat { d } \left(\mathbf{r},-\omega_{0} ;\right.\right. \\
& \left.\left.-\omega_{0}, 2 \omega_{0}\right):\left(\mathbf{e}_{\mathrm{f}}^{*} \mathbf{e}_{\mathrm{s}}\right)\right] \exp \left(-l \mathbf{G}_{0} \cdot \mathbf{r}\right)=0, \\
& \imath \frac{\partial b}{\partial z}+\frac{l}{v_{\mathrm{gs}}} \frac{\partial b}{\partial t}+a^{2} \exp (-\imath \Delta k z) \frac{\omega_{0} \varepsilon_{0}}{2 L \tilde{P}_{\mathrm{s}}} \int_{\Omega} \mathrm{d}^{3} \mathbf{r} \times \mathbf{e}_{\mathrm{s}}^{*} \cdot\left[\hat { d } \left(\mathbf{r},-2 \omega_{0} ;\right.\right. \\
& \left.\left.\omega_{0}, \omega_{0}\right):\left(\mathbf{e}_{\mathrm{f}} \mathbf{e}_{\mathrm{f}}\right)\right] \exp \left(\mathbf{G}_{0} \cdot \mathbf{r}\right)=0,
\end{aligned}
$$

where $v_{\mathrm{gf}, \mathrm{s}}=c / n_{\mathrm{gf}, \mathrm{s}}=1 /\left(\partial k_{\mathrm{f}, \mathrm{s} z} / \partial \omega\right)_{\omega_{0}}$ are the group velocities $(\mathrm{GV})$ in the $z$ direction and $\tilde{P}_{\mathrm{f}, s}=0.5 \operatorname{Re} \int \mathrm{d} A\left(\mathbf{e}_{n_{\mathrm{f}, s}} \times\right.$ $\left.\mathbf{h}_{n_{\mathrm{f}, s}}^{*}\right)_{z}$ is the modal Poynting vector flux per unit cell in propagation direction for the unperturbed system and does not depend on $z$ [49]. These equations are valid only [36] if there exists one reciprocal lattice vector $\mathbf{G}_{0}$ such that $\mathbf{k}_{\mathrm{s} \perp}-2 \mathbf{k}_{\mathrm{f} \perp}+$ $\mathbf{G}_{0 \perp}=0$ and the phase mismatch in propagation direction $\Delta k=k_{\mathrm{s} z}^{0}-2 k_{\mathrm{f} z}^{0}+G_{0 z}$ is small, i. e., if $|\Delta k| \ll 2 \pi / L$. Here we allow also for BSHG, i. e., we allow for a negative sign $\sigma=\operatorname{sgn}\left(v_{\mathrm{gs}}\right)$ of the $\mathrm{SH} \mathrm{GV}$, where consequently [50] also $\operatorname{sgn}\left(\tilde{P}_{\mathrm{s}}\right)=\sigma$.

As known from Ref. [48], for materials with Kleinman symmetry and for a real $\hat{d}$ tensor, the following relation holds

$$
\begin{aligned}
\mathbf{e}_{\mathrm{f}}^{*} \cdot & {\left[\hat{d}\left(\mathbf{r},-\omega_{0} ;-\omega_{0}, 2 \omega_{0}\right):\left(\mathbf{e}_{\mathrm{f}}^{*} \mathbf{e}_{\mathrm{s}}\right)\right] } \\
= & \mathbf{e}_{\mathrm{s}} \cdot\left[\hat{d}\left(\mathbf{r},-2 \omega_{0} ; \omega_{0}, \omega_{0}\right):\left(\mathbf{e}_{\mathrm{f}}^{*} \mathbf{e}_{\mathrm{f}}^{*}\right)\right],
\end{aligned}
$$


giving one single effective nonlinear coefficient for both equations:

$$
\begin{aligned}
& \imath \frac{\partial a}{\partial z}+\frac{\imath}{v_{\mathrm{gf}}} \frac{\partial a}{\partial t}+b a^{*} \exp (\iota \Delta k z) \frac{\omega_{0} \varepsilon_{0} \kappa}{2 L \tilde{P}_{\mathrm{f}}}=0, \\
& \iota \frac{\partial b}{\partial z}+\frac{\imath}{v_{\mathrm{gs}}} \frac{\partial b}{\partial t}+a^{2} \exp (-\imath \Delta k z) \frac{\omega_{0} \varepsilon_{0} \kappa^{*}}{2 L \tilde{P}_{\mathrm{s}}}=0 .
\end{aligned}
$$

Here $\quad \kappa=\int_{\Omega} \mathrm{d}^{3} \mathbf{r e}_{\mathrm{f}}^{*} \cdot\left[\hat{d}\left(\mathbf{r},-\omega_{0} ;-\omega_{0}, 2 \omega_{0}\right):\left(\mathbf{e}_{\mathrm{f}}^{*} \mathbf{e}_{\mathrm{s}}\right)\right] \exp$ $\left(-l \mathbf{G}_{0} \cdot \mathbf{r}\right)$. Utilizing the equivalence of energy and group velocity [50] and normalizing the fields with respect to their respective powers via $a=\left(P_{0} / \tilde{P}_{\mathrm{f}}\right)^{1 / 2} A, \quad b=$ $\left(P_{0} /\left|\tilde{P}_{\mathrm{s}}\right|\right)^{1 / 2} \exp \left(-\imath \varphi_{0}\right) B$, where $P_{0}$ is an arbitrary fixed normalization power and $\varphi_{0}$ the phase of $\kappa$ [i. e., $\kappa=$ $\left.|\kappa| \exp \left(\iota \varphi_{0}\right)\right]$, we obtain

$$
\begin{gathered}
l \frac{\partial A}{\partial z}+\frac{\imath}{v_{\mathrm{gf}}} \frac{\partial A}{\partial t}+\gamma B A^{*} \exp (\iota \Delta k z)=0, \\
\iota \sigma \frac{\partial B}{\partial z}+\frac{\iota}{\left|v_{\mathrm{gs}}\right|} \frac{\partial B}{\partial t}+\gamma A^{2} \exp (-\imath \Delta k z)=0,
\end{gathered}
$$

with

$$
\gamma=\sqrt{\frac{2 P_{0}}{\varepsilon_{0} c^{3}}} \omega_{0} \sqrt{\frac{n_{\mathrm{gf}}^{2} n_{\mathrm{gs}}}{\varepsilon_{\mathrm{nf}}^{2} \varepsilon_{\mathrm{ns}} A_{\mathrm{n}}}} .
$$

Here $\varepsilon_{\mathrm{nf}, \mathrm{s}}$ is the dielectric constant of the nonlinear material at the $\mathrm{FH}$ and $\mathrm{SH}$ frequencies, respectively, and is introduced just for comparison with the case of an unstructured material. $A_{\mathrm{n}}$ is the effective nonlinearity including the overlap of the fields and the tensorial character of the nonlinear coefficient:

$$
\begin{aligned}
& A_{\mathrm{n}} \\
& =\frac{1}{L} \frac{\varepsilon_{\mathrm{nf}}^{-2} \varepsilon_{\mathrm{ns}}^{-1}\left(\int_{\Omega} \mathrm{d}^{3} \mathbf{r} \varepsilon_{\mathrm{f}}(\mathbf{r})\left|\mathbf{e}_{\mathrm{f}}\right|^{2}\right)^{2} \int_{\Omega} \mathrm{d}^{3} \mathbf{r} \varepsilon_{\mathrm{s}}(\mathbf{r})\left|\mathbf{e}_{\mathrm{s}}\right|^{2}}{\left|\int_{\Omega} \mathrm{d}^{3} \mathbf{r e}_{\mathrm{s}}^{*} \cdot\left[\hat{d}\left(\mathbf{r},-2 \omega_{0} ; \omega_{0}, \omega_{0}\right):\left(\mathbf{e}_{\mathrm{f}} \mathbf{e}_{\mathrm{f}}\right)\right] \exp \left(\mathbf{G}_{0} \cdot \mathbf{r}\right)\right|^{2}} .
\end{aligned}
$$

Equation (13) and (14) are the same as the well-known equations describing SHG with plane waves in a homogeneous medium [2] with an additional slow time dependence.

In our physical power scaling of the equations we obtain for the transported powers per transverse unit cell $P_{\mathrm{f}}(z)=$ $P_{0}|A(z)|^{2}$ and $P_{\mathrm{S}}(z)=P_{0}|B(z)|^{2}$ for $\mathrm{FH}$ and $\mathrm{SH}$, respectively. Here it should be kept in mind that for $\sigma=-1$ the SH power is directed backward, resulting in a negative Poynting vector flux. One conserved quantity is $P_{\mathrm{f}}(z)+\sigma P_{\mathrm{s}}(z)$. In the extended zone scheme of the dispersion relation in $\mathbf{k}$ space, where $\mathbf{k}$ is not restricted to the first Brillouin zone, we have $\mathbf{G}_{0}=0$ and extended zone Bloch vectors $\mathbf{k}_{\mathrm{f}, \mathrm{s}}^{\mathrm{e}}$ with $\Delta k=k_{\mathrm{s} z}^{0 \mathrm{e}}-2 k_{\mathrm{f} z}^{0 \mathrm{e}}$ and $\mathbf{k}_{\mathrm{s} \perp}^{\mathrm{e}}-2 \mathbf{k}_{\mathrm{f} \perp}^{\mathrm{e}}=0$ are obtained instead.

In the limit of the interaction of linearly polarized plane waves in a homogeneous material we simply obtain

$$
\begin{gathered}
\gamma=\sqrt{\frac{2 P_{0}}{\varepsilon_{0} c^{3}}} \omega_{0} \frac{1}{\sqrt{\varepsilon_{\mathrm{nf}} \sqrt{\varepsilon_{\mathrm{ns}}} A_{\mathrm{n}, \mathrm{hom}}}}, \\
A_{\mathrm{n}, \mathrm{hom}}=A_{\perp}\left|d_{\mathrm{eff}}\right|^{-2}
\end{gathered}
$$

with the unit cell cross section $A_{\perp}$ and the effective nonlinear coefficient $d_{\text {eff }}$ [48]. Here, obviously the intensities are $I_{\mathrm{f}, \mathrm{s}}=P_{\mathrm{f}, \mathrm{s}} / A_{\perp}$. Applying this modal approach to a bulk medium using antiparallel plane waves and by introducing an artificial virtual period $L=2 \pi /\left|k_{\mathrm{s} z}^{0}-2 k_{\mathrm{f} z}^{0}\right|$ gives the equations for phase matched $(\Delta k=0) \mathrm{BSHG}$, which is in contradiction to previous investigations. Closer inspection, however, reveals that the nonlinear coefficient vanishes in this case. One possibility of optimal overlap at simultaneous phase matching for BSHG would be using a (homogeneous on the wavelength scale) left-handed material (LHM) [51-54] with the appropriate material dispersion, as LHMs are intrinsically dispersive.

For the stationary $(\partial A / \partial t=\partial B / \partial t=0)$, forward $(\sigma=1)$ phase matched $(\Delta k=0)$ case without $\mathrm{SH}$ input and with $A(0)=A_{0}$ we get the solution [2] $A(z)=A_{0} \operatorname{sech}\left(\gamma\left|A_{0}\right| z\right)$, $B(z)=\imath A_{0}^{2} \tanh \left(\gamma\left|A_{0}\right| z\right) /\left|A_{0}\right|$. For a fundamental power input $P_{\mathrm{f}}(0)=P_{0}$, i. e., $\left|A_{0}\right|=1$, we obtain for the actual powers per unit cell

$$
\begin{aligned}
& P_{\mathrm{f}}(z)=P_{0} \operatorname{sech}^{2}(\gamma z), \\
& P_{\mathrm{s}}(z)=P_{0} \tanh ^{2}(\gamma z),
\end{aligned}
$$

where $\gamma \propto \sqrt{P_{0}}$ as given in Eq. (15).

For the stationary backward $(\sigma=-1)$ phase matched case the analysis was performed considerably later [6]. Here the boundary conditions for the $\mathrm{FH}$ and $\mathrm{SH}$ are different, as the $\mathrm{SH}$ is propagating (energy transport) in the negative $z$ direction. Therefore, we have to fix the length of the nonlinear medium and to impose at this exit boundary the condition that there is no $\mathrm{SH}$ input from that direction. In the following $l$ is the length of the nonlinear part of the PhC. The linear properties continue (infinitely) after that length as otherwise this interface would lead to linear reflections. The solution for the amplitudes is now $A(z)=A_{l} \sec \left[\gamma\left|A_{l}\right|(z-l)\right]$, $B(z)=-l A_{l}^{2} \tan \left[\gamma\left|A_{l}\right|(z-l)\right] /\left|A_{l}\right|$. Here, $A_{l}=A(l)$ is the $\mathrm{FH}$ amplitude at the end of the nonlinear medium and is connected to the amplitude at the FH input facet $A_{0}=A(0)$ via the transcendental equation $A_{l}=A_{0} \cos \left(\gamma l\left|A_{l}\right|\right)$. For any value of $\gamma l$ there is always a solution of this equation that satisfies $\gamma l\left|A_{l}\right|<\pi / 2$ leading to $|A(z)|$ and $|B(z)|$ monotonically decreasing with $z$ in the nonlinear region. Additional solutions appearing for $\gamma l\left|A_{0}\right|$ larger than the critical value of $6.202395 \ldots$ lead to intermediate singularities of $\sec \left[\gamma\left|A_{l}\right|(z-l)\right]$ and $\tan \left[\gamma\left|A_{l}\right|(z-l)\right]$ upon propagation and, hence, are unphysical. For a FH input power of $P_{0}$, i.e., $\left|A_{0}\right|=1$, the powers of the waves per unit cell are given by

$$
\begin{aligned}
& P_{\mathrm{f}}(z)=P_{0}\left|A_{l}\right|^{2} \sec ^{2}\left[\gamma\left|A_{l}\right|(z-l)\right], \\
& P_{\mathrm{s}}(z)=P_{0}\left|A_{l}\right|^{2} \tan ^{2}\left[\gamma\left|A_{l}\right|(z-l)\right],
\end{aligned}
$$

where $\left|A_{l}\right|$ is obtained from $\left|A_{l}\right|=\cos \left(\gamma\left|A_{l}\right| l\right)$.

For large phase mismatch $|\Delta k| \gg \gamma\left|A_{0}\right|$ and because due to $|A(0)|=1$ and $B(0)=0$ also the prerequisite $|A(0)| \gg|B(0)|$ is fulfilled, we may resort to the UDPA [2] $\left(P_{\mathrm{f}}(z)=P_{0}=\right.$ const.) to obtain the solutions

$P_{\mathrm{s}}(z)=P_{0} \gamma^{2} \times\left\{\begin{array}{cl}z^{2} \operatorname{sinc}^{2}\left(\frac{\Delta k}{2} z\right) & \text { forward SHG } \\ (z-l)^{2} \operatorname{sinc}^{2}\left[\frac{\Delta k}{2}(z-l)\right] & \text { BSHG. }\end{array}\right.$

Large values of $\left|A_{l}\right|$ for large normalized phase mismatch $\Delta k l$ lead to an instability of the solution [17]. In the following we work in the regime of small pump values.

For characterizing the strength of the nonlinear interaction in the $\mathrm{PhC}$ we compare the SHG efficiencies defined by 
$\eta=P_{\mathrm{s}}(z) / P_{\mathrm{f}}^{2}(0) z^{2}$ in the UDPA, which is also valid for $\Delta k=0$ for small propagation distances $z$. Because in the backward case the $\mathrm{SH}$ grows from $l$ into negative direction, $z$ has to be replaced by $(z-l)$ in this expression. We find

$$
\eta=2 \frac{\omega_{0}^{2}}{\varepsilon_{0} c^{3} \varepsilon_{\mathrm{f}} \sqrt{\varepsilon_{\mathrm{s}}}}\left\{\begin{array}{cc}
\frac{d_{\mathrm{eff}}^{2}}{A_{\perp}} & \text { bulk } \\
\frac{n_{\mathrm{gf}}^{2} n_{\mathrm{gs}}}{\varepsilon_{\mathrm{f}} \sqrt{\varepsilon_{\mathrm{s}}}} \frac{1}{A_{n}} & \text { PhC. }
\end{array}\right.
$$

We define the nonlinear FOM as ratio of the $\mathrm{PhC}$ efficiency and the bulk efficiency (at the same pump frequency $\omega_{0}$ ) to obtain

$$
\mathrm{FOM}=\frac{n_{\mathrm{gf}}^{2} n_{\mathrm{gs}}}{\varepsilon_{\mathrm{f}} \sqrt{\varepsilon_{\mathrm{s}}}} \frac{d_{\mathrm{eff}, \mathrm{bulk}}^{-2} A_{\perp}}{A_{\mathrm{n}}} .
$$

For the bulk case we assumed here that phase and group velocities do not differ substantially as we consider only weakly dispersive materials. If QPM is used in the bulk case the FOM has to be multiplied by a factor of $(\pi / 2)^{2}$. Obviously, $A_{\mathrm{n}}$ corresponds to an effective area, incorporating the $\hat{d}$ tensor already. Usually, the second factor of the right-hand side of Eq. (25) will be always smaller than unity as the $\mathrm{PhC}$ comprises also air. However, the first factor can overcompensate this effect and even dramatically enhance the FOM when the group velocities of the interacting Bloch waves become substantially smaller than the phase velocities in the bulk medium, as it happens, e.g., close to band edges. This effect will be verified in the following sections.

\section{PHASE MATCHING FOR FORWARD AND BACKWARD SHG}

Currently, 2D PhC membranes of $\mathrm{LiNbO}_{3}$ are expected to represent a novel experimental platform for nonlinear experiments [33]. Here the membrane provides the vertical guidance, whereas the in-plane dynamics is essentially governed by the $2 \mathrm{D} \mathrm{PhC}$ structure. Therefore in the following we restrict ourselves to a true 2D system, where $y$ is the invariant direction of the system perpendicular to the plane of periodicity. We use a hexagonal lattice of circular air holes with hole radius $r$ and lattice pitch $a$ and neglect material dispersion and anisotropy. However, although the dispersion provided by the periodic structure is much stronger the material dispersion and anisotropy have finally to be taken into account for the proper designs of experiments. This can be mimicked via a small correction of the hole radius as was shown in Ref. [36]. This should be kept in mind for obtaining proper designs for experiments. We choose an (isotropic) dielectric constant of $\mathrm{LiNbO}_{3}$ of $\varepsilon=2.1588^{2}$ corresponding to the extraordinary wave of the in reality isotropic material at a wavelength of $1 \mu \mathrm{m}$ and room temperature [55]. A ratio $r / a=0.3$ is used and the dispersion relation of the $\mathrm{PhC}$ is calculated solving Maxwell's equations with periodic boundary conditions by preconditioned conjugate-gradient minimization of the block Rayleigh quotient in a plane wave basis, using a freely available software package [56]. We focus on TE-polarized light first.

\section{A. Efficiency enhancement of forward SHG}

From the conventional band structure diagram, where the dispersion relation along high-symmetry lines is depicted

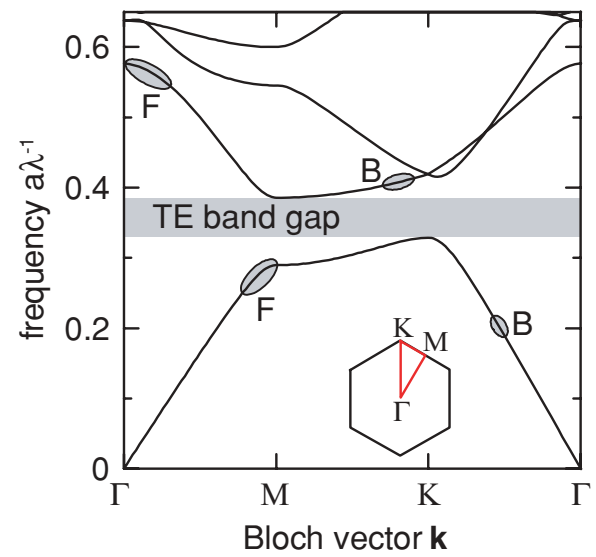

FIG. 1. (Color online) TE band structure diagram for the PhC with $r / a=0.3$ and $\varepsilon=2.1588^{2}$. The gray ellipses mark the regions used for phase matching in the forward (F) and backward (B) cases.

(see Fig. 1) we can identify the TE photonic bandgap. For visualization of the phase matching condition $\Delta k=0$, which is equivalent to $2 \omega_{n_{\mathrm{f}}}\left(\mathbf{k}_{\mathrm{s}}^{\mathrm{e}} / 2\right)=\omega_{n_{\mathrm{s}}}\left(\mathbf{k}_{\mathrm{s}}^{\mathrm{e}}\right)$, the functions $\omega_{n_{\mathrm{f}}}\left(\mathbf{k}_{\mathrm{s}}^{\mathrm{e}} / 2\right)$ and $\omega_{n_{\mathrm{s}}}\left(\mathbf{k}_{\mathrm{s}}^{\mathrm{e}}\right)$ are plotted in the extended zone scheme in the isofrequency diagram in Fig. 2 together with the collinear phase matching curve $2 \omega_{n_{\mathrm{f}}}\left(\mathbf{k}_{\mathrm{s}}^{\mathrm{e}} / 2\right)=\omega_{n_{\mathrm{s}}}\left(\mathbf{k}_{\mathrm{s}}^{\mathrm{e}}\right)$. The frequencies are given in terms of the normalized frequency $u=\omega a / 2 \pi c=$ $a / \lambda$.

It can be seen that for any direction of the (extended zone) Bloch vectors phase matching can be achieved. However, for meaningful operation in reality the directions of the energy flow (corresponding to the group velocity directions, see Ref. [50]) of both waves should only slightly differ. Then here, the two high-symmetry directions $\Gamma \mathrm{M}$ und $\Gamma \mathrm{K}$ are obviously

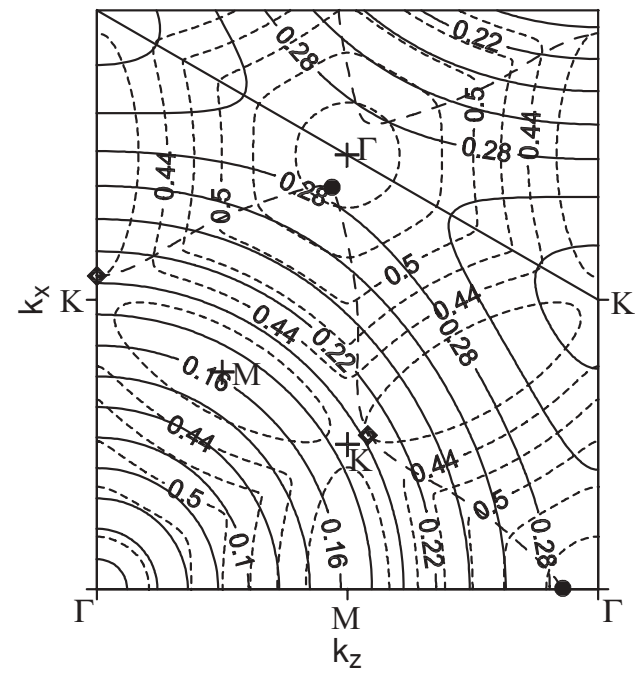

FIG. 2. Isofrequency curves for the hexagonal PhC lattice with $r / a=0.3$ and TE-polarized light. Band 1 (solid curves) is scaled by a factor of 2 [i.e., $\omega_{1}(\mathbf{k} / 2)$ ], the short-dashed curves correspond to (the unscaled) band 2 . The long-dashed curve represents perfect phase matching for collinear propagation, the black dots mark the points of collinear phase matching in (two of six) equivalent $\Gamma \mathrm{M}$ directions at $u_{\mathrm{f}}=0.28353$, the diamond symbols mark the points of phase matching for BSHG in two equivalent $\Gamma \mathrm{K}$ directions at $u_{\mathrm{f}}=0.20491$. For BSHG $k_{x}$ and $k_{z}$ are exchanged. 
ideal. A closer inspection of Fig. 2 reveals phase matched forward SHG in $\Gamma M$ direction (at $u_{\mathrm{f}}=0.28353$ ) and phase matched BSHG in $\Gamma$ K direction (at $u_{\mathrm{f}}=0.20491$ ).

In the forward case the FH field distribution still resembles a plane wave, with a strong standing-wave character, though, with the transverse electric field component $e_{\mathrm{f} x}$ being the major component. Also the SH field is mainly polarized in the transverse direction. Hence, in order to utilize the largest and diagonal $\hat{d}$ tensor component $d_{33}$ we orient the nonlinear crystal's $c$ axis along $x$ in the laboratory frame. Simultaneously the remaining much smaller components of the nonlinear tensor may be neglected. Then we obtain from (16) $A_{\mathrm{n}}=A_{\text {eff }} /\left|d_{33}\right|^{2}$ with the effective area for this second-order interaction. In the $2 \mathrm{D}$ system the respective effective width amounts to $A_{\text {eff }}=3.7 a$, which means a 3.7fold smaller field overlap compared to the bulk case. On the other hand, the group indices are $n_{\mathrm{gf}}=3.8$ and $n_{\mathrm{gs}}=5.2$, leading in total to a nonlinear FOM of 2. However, this FOM corresponds to the case of perfect phase matching in a plane wave configuration utilizing likewise the large $d_{33}$ coefficient which is unrealistic. It can only be exploited in QPM geometries, which leads then to a FOM of 4.9.

In the backward case (propagation in $\Gamma \mathrm{K}$ direction) the situation is slightly different. The FH fields resemble even more a traveling plane wave, as the point of phase matching is far away even from the fundamental Bragg resonances (see Fig. 1). The second-harmonic fields, however, have a standing wavelike pattern in the transverse direction. In particular, due to its symmetry the transverse component leads to an exactly vanishing nonlinear effective coefficient. The longitudinal component still gives a nonvanishing contribution of $A_{\text {eff }}=$ $1350 a$, which is very large. This value is associated with the tensor component $d_{33}$, and with the group indices $n_{\mathrm{gf}}=1.8$ and $n_{\mathrm{gs}}=-6.1$, resulting in FOM $=0.0015$. Exploiting the transverse component of the fundamental field, however, one obtains a much smaller effective width of $A_{\text {eff }}=18 a$, associated with $d_{31}$. In fact, here the nondiagonal coefficient $d_{31}$ generating a polarization component in propagation direction from the transverse electric field component $e_{x}$ provides a doubled contribution to the effective nonlinear coefficient, although $d_{33} \approx 6 d_{31}$.

\section{B. Giant backward SHG enhancement by slow light}

The nonlinear FOM of 0.0015 in the investigated 2D PhC is still larger than for most other approaches but means a low efficiency compared to the forward case. Therefore in the following we want to improve the FOM. Obviously, there are two possibilities. First, increasing the field overlap and, hence, decreasing the effective area and, second, using slow light for both waves. In our hexagonal structure with TE polarization we could not identify regions with field distributions without transverse knots and good overlap which at the same time allow for phase matching with a backward wave. One reason may be the photonic bandgap, which tends to shift the higher-order bands $(2 \cdots 6)$ to even higher frequencies. This way, the usual (1D-like) Bragg resonances at the high-symmetry points $\Gamma, \mathrm{M}$, and $\mathrm{K}$, which in a simple $1 \mathrm{D}$ picture correspond to two standing waves separated by a small gap induced by the 1D periodicity are also shifted up more. This allows

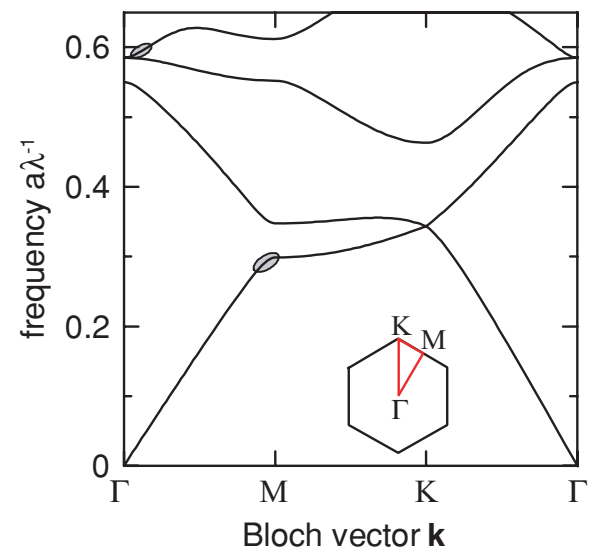

FIG. 3. (Color online) Band diagram for the hexagonal PhC lattice with $r / a=0.35$ and TM-polarized light. The gray ellipses mark the regions used for phase matching for backward SHG.

a phase matched combination of the first band with only the second band (the lower egde of the small stop gap at the $\Gamma$ point), which is usually a forward wave, due to the restriction $\mathbf{k}_{\mathrm{s}}^{\mathrm{e}}=2 \mathbf{k}_{\mathrm{f}}^{\mathrm{e}}$. The only possible phase matching is then to modes with transverse knots, as shown earlier here. Consequently, we investigate the TM polarization now, as there is no photonic bandgap. Thus the $2 \mathrm{D}$ periodicity could even shift the resonances to smaller frequencies and allow for phase matching to the backward wave that still resembles a plane wave. We find phase matching for $r / a=0.35$ at $u_{\mathrm{f}}=0.29544$ (see Figs. 3 and 4) with the group indices $n_{\mathrm{gf}}=4$ (first band) and $n_{\mathrm{gs}}=-4.9$ (fourth band).

As $e_{y}$ is the only electric field component in a TM configuration it is optimal to orient the $\mathrm{LiNbO}_{3} c$ axis along the $y$ direction (perpendicular to the plane of periodicity). We obtain from the fields a good overlap with $A_{\text {eff }}=5 a$ and finally

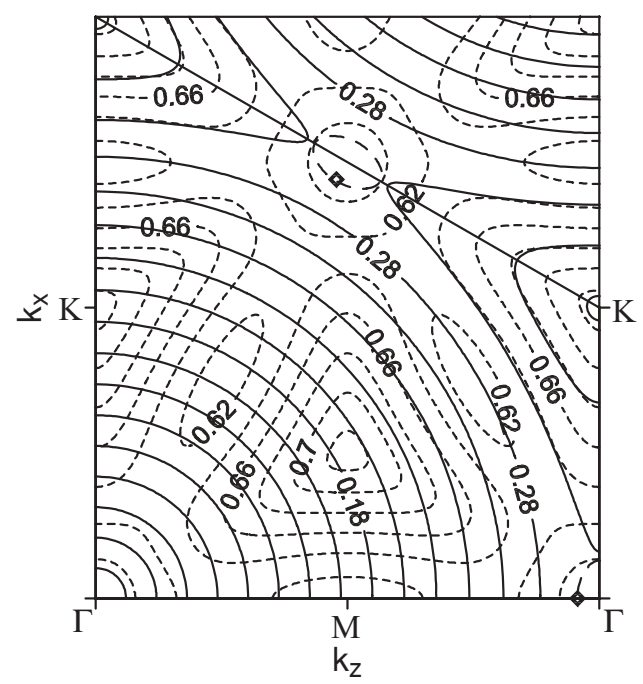

FIG. 4. Isofrequency curves for the same structure as in Fig. 3. Band 1 (solid curves) is scaled by a factor of 2 [i.e., $\omega_{1}(\mathbf{k} / 2)$ ], the short-dashed curves correspond to (the unscaled) band 4 . The long-dashed curve represents perfect phase matching for collinear propagation, the diamond symbols mark the points of collinear phase matching for BSHG in $\Gamma \mathrm{M}$ direction at $u_{\mathrm{f}}=0.29544$. 
$\mathrm{FOM}=1.5$ (QPM FOM of 3.8) which is only slightly smaller than for the TE forward case.

\section{PROPAGATION SIMULATIONS WITH THE NONLINEAR FINITE-DIFFERENCE TIME-DOMAIN METHOD}

The advances in the performance of computer hardware enabled for direct numerical treatment of microsized photonic components. We model the system using the FDTD method [57], where Maxwell's equations are discretized in time and space without further approximation. This treatment ensures that all effects of light propagation, e.g., field discontinuity at high-contrast dielectric interfaces, are properly accounted for. Moreover, the limits of frequently used approximations, e.g., the UDPA [34,39,40] can be identified. The quadratically nonlinear polarization of $\mathrm{LiNbO}_{3}$ in the time domain used here [cf. Eqs. (6) and (7)] is given by

$$
\mathbf{P}_{\mathrm{nl}}(\mathbf{r}, t)=2 \varepsilon_{0} d_{33}\left(-2 \omega_{0} ; \omega_{0}, \omega_{0}\right) E_{x, y, z}^{2}(\mathbf{r}, t) \mathbf{n}_{x, y, z},
$$

where $E_{x, y, z}$ is the (real) $x, y, z$ component of the electric field in the time domain, depending on whether we are treating the TE forward $(x)$, TE backward $(z)$, or TM (y) case. $\mathbf{n}_{x, y, z}$ is the corresponding unit direction vector. Equation (26) expresses an instantaneous nonlinear response to the exciting electric field. Only the tensor component $d_{33}$ (oriented along $x, y, z$ in the laboratory frame) is taken into account and the others are neglected. For reasons of numerical stability, the nonlinear term is treated implicitly in the usually explicit FDTD scheme. However, due to the simpler form of Eq. (26) we solve the nonlinear equations analytically instead of using an iterative algorithm [58]. As in the previous section in the following we neglect material dispersion and anisotropy of $\mathrm{LiNbO}_{3}$, although their incorporation is not a fundamental problem. However, taking the material dispersion into account would increase the computing time considerably.

As the phase mismatch is essentially determined by the phase evolution of the linear modes it is important to accurately model the phase over the whole propagation length, i.e., the discretization dependent numerical dispersion should be substantially lower than the "true" physical dispersion over the $\mathrm{PhC}$ length [59]. Here we aim at propagation lengths of up to 700 lattice periods corresponding to a length of the order of $1 \mathrm{~mm}$ with pump frequencies in the near infrared. From careful examination of SHG in a homogeneous medium we found a spatial discretization of $\Delta x=\lambda_{\mathrm{f}} / 424$ to be sufficient for the investigated propagation lengths. The time step was taken to be $\Delta t=\Delta x / 2 c$, relatively close to the (Courant) criterion for numerical stability [57] in order not to introduce too much additional numerical dispersion.

We use periodic boundary conditions in the direction perpendicular to the propagation direction to reduce numerical efforts. Consequently, for propagation in $\Gamma \mathrm{M}$ direction the transverse size of the computational domain is $a$ and the periodicity along $z$ is $\sqrt{3} a$, corresponding to the conventional (rectangular) elementary cell of the hexagonal lattice. In propagation direction the computing window was terminated by perfectly matched layer boundaries [57]. As excitation a plane wave of adjustable intensity and frequency was used at one end, in a short unstructured region before the $\mathrm{PhC}$ facet in the case of forward SHG, thus approximating wide beams by Bloch waves. Otherwise the $\mathrm{PhC}$ was continued into the boundaries to lower reflections of backward propagating light. After a steady state was reached discrete Fourier transforms at the $\mathrm{FH}$ and $\mathrm{SH}$ frequency were applied to the fields at every grid point, giving the corresponding (complex) fields in the Fourier domain. From this, all interesting quantities, as the Poynting vector or flux along propagation direction, can be obtained. To obtain the time-averaged Poynting vector flux of FH and $\mathrm{SH}$ waves, corresponding to the directed power, we integrate the real part of the $z$ component of the complex Poynting vector over the cross section (along $x$ ) at every $z$. To be able to compare the efficiencies for different frequency detunings the input power at the very beginning of the $\mathrm{PhC}$ was kept constant for all calculations. A constant sum (or difference) of $\mathrm{FH}$ and $\mathrm{SH}$ powers along propagation confirms energy conservation and that almost no energy is transferred to other frequency harmonics. For a meaningful comparison of the efficiencies of different configurations with different transverse unit cell size we actually give the power per transverse unit cell normalized to the cross-section area of this unit cell, corresponding to the average intensity of the light and to the conventional, transversely constant, intensity of a plane wave. Moreover, as the SHG efficiency explicitly depends on the pump frequency $\left(\gamma \propto \omega_{0}\right)$, we have to compare the values along propagation in the three different systems for the same frequency, resulting in different lattice periods $a_{1}, a_{2}$, and $a_{3}$, where case 1 is the TE forward SHG, case 2 is the TE BSHG, and 3 the TM BSHG.

In Fig. 5 the dependence of the $\mathrm{FH}$ and $\mathrm{SH}$ average intensities on $z$ for phase matched forward SHG with $r / a=$ 0.3 and for plane waves in a nondispersive bulk medium, which is phase matched automatically, is displayed.

The aperiodic $\tanh ^{2}(z)$ behavior of the SH can be identified. However, in the $\mathrm{PhC}$ the $\mathrm{SH}$ intensity grows much faster. From these two curves a FOM of 2.3 is obtained which

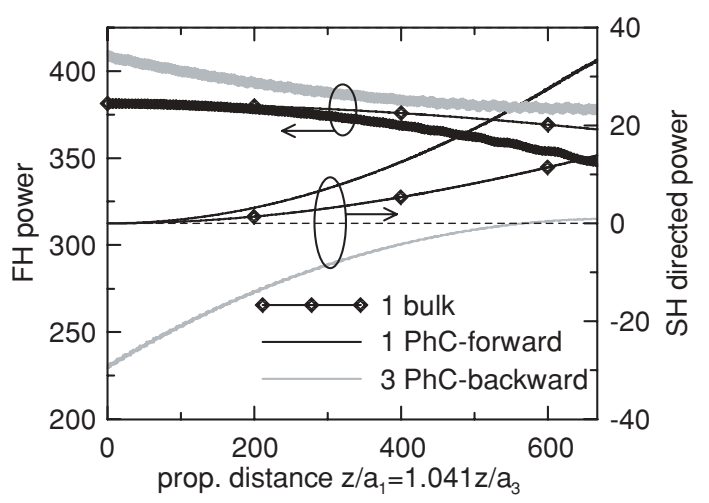

FIG. 5. FH and SH average intensities for phase matched SHG in the $\mathrm{PhC}$ with $r / a=0.3$, for the phase matched bulk at $u_{\mathrm{f}}=0.28369$ and for TM BSHG in the PhC with $r / a=0.35$. The same units were used for all power values. 


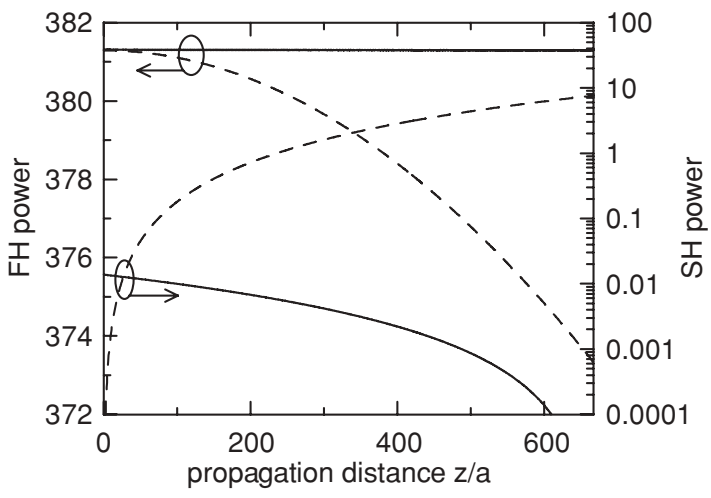

FIG. 6. Average FH and $\mathrm{SH}$ intensities for phase matched BSHG with TE-polarized light in the PhC structure of Fig. 1 for propagation in the $\Gamma \mathrm{K}$ direction (solid lines) and for phase matched SHG in the bulk (dashed lines). The same intensity units were used as in Fig. 5. The $\mathrm{SH}$ is shown on a logarithmic scale.

is in excellent agreement with the value obtained from the modal method. Also, for the detuned pump frequencies, the oscillation periods for different signs and magnitudes of the resulting phase mismatch are in perfect agreement with the modal method [36].

Next, we investigate the BSHG for the very same structure with the same orientation of $d_{33}$ but for propagation in $\Gamma \mathrm{K}$ direction and at different frequencies. In Fig. 6 we can see the dependence of the average $\mathrm{FH}$ and $\mathrm{SH}$ intensities on propagation distance. The dramatically decreased efficiency with a FOM of only 0.0017 can clearly be seen. This again is in very good agreement with the value obtained from the modal approach. For this FH power at this propagation distance even the UDPA is valid. For appropriately ( 25 times) increased FH input average intensity we observe a noticeable depletion of the pump.

Finally, in the TM structure we again obtain a similarly high conversion efficiency as for the forward case (see Fig. 5) as predicted from the modal approach. To be able to compare the powers and to directly obtain the FOM from the graph, the FH power at the exit $z=l$ was adjusted to match the input powers of the forward calculations. We can also see the oscillations for the phase mismatched case shown in Fig. 7. One difference to the forward case is the strongly reduced frequency bandwidth of the phase matching conditions. We estimate a bandwidth smaller by a factor of more than 10 . The reduction is clear from the modal description [12]. As two dispersion relations with opposite slope (opposite signs of the group velocity) cross, $|\Delta k|$ increases rapidly for small changes of the frequency. This is in contrast to the forward case. Essentially, in a first-order approximation, the phase matching frequency bandwidth is inversely proportional to the difference of the inverse group velocities [12]. In the slow light regime close to the band edge, however, this is a crude approximation, as the dispersion curve cannot be well approximated by a linear behavior.

A weak forward-propagating SH field can be identified. It becomes manifest in a constant but nonzero SH output at the end of the crystal and in a shift of the behavior

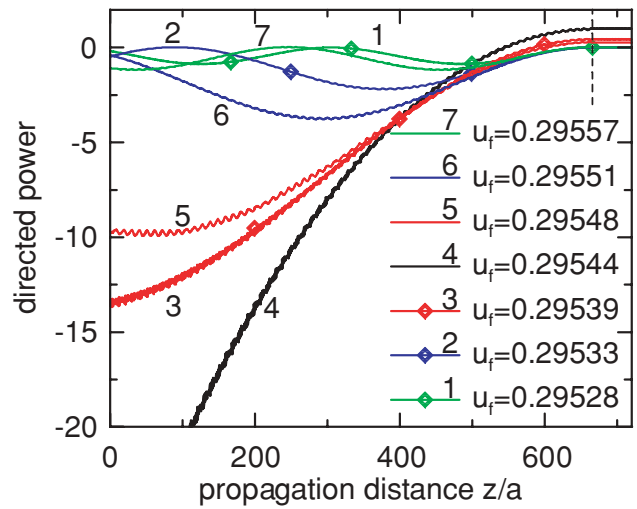

FIG. 7. (Color online) Average directed SH intensities for BSHG with TM polarized in the $\mathrm{PhC}$ structure of Fig. 3 for propagation in the $\Gamma \mathrm{M}$ direction for different pump frequencies. Line 4 corresponds to the frequency of phase matching. The same intensity units were used as in Fig. 5. The vertical dashed line marks the end of the nonlinear material.

$\propto-\sin ^{2}\left[\pi(z-l) / 2 L_{\mathrm{c}}\right]$ of the Poynting vector flux to slightly positive values where it should be 0 . This is caused by reflections of the backward generated $\mathrm{SH}$ at the input interface where the $\mathrm{PhC}$ starts. Therefore, this effect is more pronounced for oscillation periods (corresponding to $2 L_{\mathrm{c}}=2 \pi /|\Delta k|$, and hence, depending on the phase mismatch) where a backward power maximum hits this interface and is largest for the phase matched case but vanishes if a minimum coincides with the front interface. The reflections are relatively large due to the interface between modes of so different group velocities (slow and normal light). However, due to the very large phase mismatch to the forward propagating $\mathrm{FH}$ wave these forward $\mathrm{SH}$ waves virtually do not contribute to the nonlinear interaction. Comparing the backward growth at the end of the crystal with the phase matched bulk we obtain in FOM of 1.8, which again is in very good agreement with the value proposed by the modal method.

The efficiency in the phase matched case can be even further increased by shifting the point of phase match still further into direction of the high symmetry points $\Gamma$ and $\mathrm{M}$ by increasing the hole radius. This effect, however, is much less pronounced than in the TE forward case.

\section{CONCLUSIONS}

We have investigated both forward and backward type I SHG of wide beams in 2D PhCs. We propose a structure where forward SHG and BSHG are phase matched. The introduced nonlinear FOM allows for an easy comparison of the efficiency with the phase matched bulk case. Using slow light Bloch modes for the FH and SH waves we have obtained a greatly enhanced SHG efficiency. For fixed input power per transverse unit cell (corresponding to the beam intensity) the slow light leads to much larger field amplitudes and, hence, largely enhances the strength of the nonlinear interaction. For forward SHG in an ideal structure an enhancement of more than 60 can be obtained (see Ref. [36]). We have suggested a design for BSHG that allows an enhancement compared to the QPM bulk case of almost 3, which is very high for a 
backward configuration. With the current state of the art in nanotechnology, $\mathrm{PhCs}$ are promising candidates for achieving larger SHG efficiencies than with QPM for the backward case. The quantitative analysis based on a modal approach is in excellent agreement with results obtained from the nonlinear FDTD.

\section{ACKNOWLEDGMENTS}

The authors gratefully acknowledge financial support from the German Federal Ministry of Education and Research (Innoregio, ZIK), the Deutsche Forschungsgemeinschaft (DFG, research unit 532), and the Australian Research Council.
[1] P. A. Franken, A. E. Hill, C. W. Peters, and G. Weinreich, Phys. Rev. Lett. 7, 118 (1961).

[2] J. A. Armstrong, N. Bloembergen, J. Ducuing, and P. S. Pershan, Phys. Rev. 127, 1918 (1962).

[3] P. A. Franken and J. F. Ward, Rev. Mod. Phys. 35, 23 (1963).

[4] D. S. Hum and M. M. Fejer, C. R. Phys. 8, 180 (2007), and references therein.

[5] S. E. Harris, Appl. Phys. Lett. 9, 114 (1966).

[6] P. Russell, IEEE J. Quantum Electron. 27, 830 (1991).

[7] M. Fejer, G. Magel, D. Jundt, and R. Byer, IEEE J. Quantum Electron. 28, 2631 (1992).

[8] Y. J. Ding and J. B. Khurgin, Opt. Lett. 21, 1445 (1996).

[9] J. U. Kang, Y. J. Ding, W. K. Burns, and J. S. Melinger, Opt. Lett. 22, 862 (1997).

[10] X. Mu, I. B. Zotova, Y. J. Ding, and W. P. Risk, Opt. Commun. 181, 153 (2000).

[11] Y. J. Ding and J. B. Khurgin, IEEE J. Quantum Electron. 32, 1574 (1996).

[12] Y. J. Ding, J. U. Kang, and J. B. Khurgin, IEEE J. Quantum Electron. 34, 966 (1998).

[13] C. Canalias and V. Pasiskevicius, Nat. Photon. 1, 459 (2007).

[14] C. Conti, S. Trillo, and G. Assanto, Phys. Rev. Lett. 85, 2502 (2000).

[15] M. Conforti, C. de Angelis, U. K. Sapaev, and G. Assanto, Opt. Express 16, 2115 (2008).

[16] G. D'Alessandro, P. St. J. Russell, and A. A. Wheeler, Phys. Rev. A 55, 3211 (1997).

[17] M. Conforti, A. Locatelli, C. D. Angelis, A. Parini, G. Bellanca, and S. Trillo, J. Opt. Soc. Am. B 22, 2178 (2005).

[18] N. Bloembergen and A. J. Sievers, Appl. Phys. Lett. 17, 483 (1970).

[19] A. Yariv and P. Yeh, J. Opt. Soc. Am. 67, 438 (1977).

[20] C. L. Tang and P. P. Bey, IEEE J. Quantum Electron. 9, 9 (1973).

[21] J. P. van der Ziel and M. Ilegems, Appl. Phys. Lett. 28, 437 (1976).

[22] J. Martorell and R. Corbalán, Opt. Commun. 108, 319 (1994).

[23] M. Scalora, M. J. Bloemer, A. S. Manka, J. P. Dowling, C. M. Bowden, R. Viswanathan, and J. W. Haus, Phys. Rev. A 56, 3166 (1997).

[24] M. Bertolotti, J. Opt. A: Pure Appl. Opt. 8, S9 (2006), and references therein.

[25] N. Mattiucci, G. D’Aguanno, M. Scalora, and M. J. Bloemer, J. Opt. Soc. Am. B 24, 877 (2007).

[26] A. Yariv, Y. Xu, R. K. Lee, and A. Scherer, Opt. Lett. 24, 711 (1999).

[27] N. A. R. Bhat and J. E. Sipe, Phys. Rev. E 64, 056604 (2001).

[28] M. Soljačić, S. G. Johnson, S. Fan, M. Ibanescu, E. Ippen, and J. D. Joannopoulos, J. Opt. Soc. Am. B 19, 2052 (2002).
[29] J. F. McMillan, X. Yang, N. C. Panoiu, R. M. Osgood, and C. W. Wong, Opt. Lett. 31, 1235 (2006).

[30] B. Corcoran, C. Monat, C. Grillet, D. J. Moss, B. J. Eggleton, T. P. White, L. O'Faolain, and T. F. Krauss, Nat. Phot. 3, 206 (2009).

[31] F. Lacour, N. Courjal, M.-P. Bernal, A. Sabac, C. Bainier, and M. Spajer, Opt. Mater. 27, 1421 (2005).

[32] M.-P. Bernal, N. Courjal, J. Amet, M. Roussey, and C. Hou, Opt. Commun. 265, 180 (2006).

[33] F. Schrempel, T. Gischkat, H. Hartung, T. Höche, E.-B. Kley, A. Tünnermann, and W. Wesch, Opt. Lett. 34, 1426 (2009).

[34] K. Sakoda and K. Ohtaka, Phys. Rev. B 54, 5742 (1996).

[35] L. Tkeshelashvili and K. Busch, Appl. Phys. B 81, 225 (2005).

[36] R. Iliew, C. Etrich, T. Pertsch, and F. Lederer, Phys. Rev. B 77, 115124 (2008).

[37] A. R. Cowan and J. F. Young, Phys. Rev. B 65, 085106 (2002).

[38] D. Coquillat, G. Vecchi, C. Comaschi, A. M. Malvezzi, J. Torres, and M. L. V. d'Yerville, Appl. Phys. Lett. 87, 101106 (2005).

[39] E. Centeno, D. Felbacq, and D. Cassagne, Phys. Rev. Lett. 98, 263903 (2007).

[40] C. Ciracì and E. Centeno, Phys. Rev. Lett. 103, 063901 (2009).

[41] C. Nistor, C. Cojocaru, Y. Loiko, J. Trull, R. Herrero, and K. Staliunas, Phys. Rev. A 78, 053818 (2008).

[42] A. Di Falco, C. Conti, and G. Assanto, Opt. Lett. 30, 1174 (2005).

[43] A. Di Falco, C. Conti, and G. Assanto, Opt. Lett. 31, 250 (2006).

[44] M. W. McCutcheon, J. F. Young, G. W. Rieger, D. Dalacu, S. Frédérick, P. J. Poole, and R. L. Williams, Phys. Rev. B 76, 245104 (2007).

[45] R. Iliew, C. Etrich, U. Peschel, and F. Lederer, IEEE J. Sel. Top. Quantum Electron. 12, 377 (2006).

[46] R. Iliew, C. Etrich, M. Augustin, E.-B. Kley, S. Nolte, A. Tünnermann, and F. Lederer, Phys. Status Solidi A 204, 3689 (2007).

[47] A. W. Snyder and J. D. Love, Optical Waveguide Theory (Chapman and Hall Ltd., London, 1983).

[48] G. D. Boyd and D. A. Kleinman, J. Appl. Phys. 39, 3597 (1968).

[49] D. Michaelis, U. Peschel, C. Wächter, and A. Bräuer, Phys. Rev. E 68, 065601(R) (2003).

[50] P. Yeh, J. Opt. Soc. Am. 69, 742 (1979). 
[51] V. M. Agranovich, Y. R. Shen, R. H. Baughman, and A. A. Zakhidov, Phys. Rev. B 69, 165112 (2004).

[52] M. Scalora, G. D'Aguanno, M. Bloemer, M. Centini, D. de Ceglia, N. Mattiucci, and Y. S. Kivshar, Opt. Express 14, 4746 (2006).

[53] I. V. Shadrivov, A. A. Zharov, and Y. S. Kivshar, J. Opt. Soc. Am. B 23, 529 (2006).

[54] A. K. Popov, V. V. Slabko, and V. M. Shalaev, Las. Phys. Lett. 3, 293 (2006).

[55] M. V. Hobden and J. Warner, Phys. Lett. 22, 243 (1966).
[56] S. G. Johnson and J. D. Joannopoulos, Opt. Express 8, 173 (2001).

[57] A. Taflove and S. C. Hagness, Computational Electrodynamics: The Finite-Difference Time-Domain Method (Artech House, Boston, MA, 2000).

[58] C. M. Reinke, A. Jafarpour, B. Momeni, M. Soltani, S. Khorasani, A. Adibi, Y. Xu, and R. K. Lee, J. Lightwave Technol. 24, 624 (2006).

[59] M. Lauritano, M. Girotto, G. Bellanca, and S. Trillo, Opt. Quantum Electron. 38, 827 (2006). 